\section{P1-S2.04 IMPACT OF FEMALE SEX WORKER TURNOVER ON HIV PREVALENCE, INCIDENCE AND CORE GROUP INTERVENTION IMPACT IN COTONOU (BENIN)}

doi:10.1136/sextrans-2011-050108.61

\section{C Boily. Imperial College, London, UK}

Background The influence of turnover of female sex workers (FSW), resulting from different durations spent in sex work or migration patterns remains partly understood but is believed to be an important determinant of HIV/STI spread among FSW and overall populations and intervention impact.

Methods A deterministic transmission dynamics model, parameterised using serial cross-sectional data, was used to simulate the course of HIV and gonorrhoea (Gc) in FSW/clients and the general population of Cotonou. Different turnover patterns were produced by varying the time spent in sex work (SW duration=lifelong to 1 year) or, equivalently, time spent in the location by migrants (sojourn duration=lifelong, 5, 1 year). Each FSW leaving the local population was replaced by a new FSW with the same risk behaviour and with FSW HIV prevalence, for the SW duration scenarios, set to that of the low risk population and, for the migration scenarios, to $0 \%, 5 \%$ or $50 \%$.

Results Shortening SW duration (or sojourn duration with initial $0 \%$ FSW HIV prevalence) from lifelong to 5- and 1-year delayed and reduced FSW HIV peak prevalence from $\sim 75 \%$ in 1999 to $56 \%$ in 2000 and $\sim 25 \%$ in 2020, respectively. The 1-year scenario increased long-term HIV prevalence of the overall female population by twofold, as the rapid turnover reduced the decline in Gc prevalence caused by AIDS differential mortality. With 1-year sojourn duration, local FSW HIV prevalence was mostly determined by the HIV prevalence of new FSW and FSW HIV incidence always exceeded prevalence (Abstract P1-S2.04 figure 1). Sudden increases in turnover of new HIV negative FSW (or sojourn duration=1 year \& $0 \%$ HIV), in a maturing epidemic (1993), could reduce FSW HIV prevalence by $>50 \%$ within 2 years but increase HIV incidence, in absence of intervention, compared to no turnover (ie, lifelong duration). The different SW and migration duration patterns did not significantly reduce the general population impact of a FSW targeted condom intervention per se, after controlling for epidemic stages, assuming similar exposure to the intervention by local and new FSW.

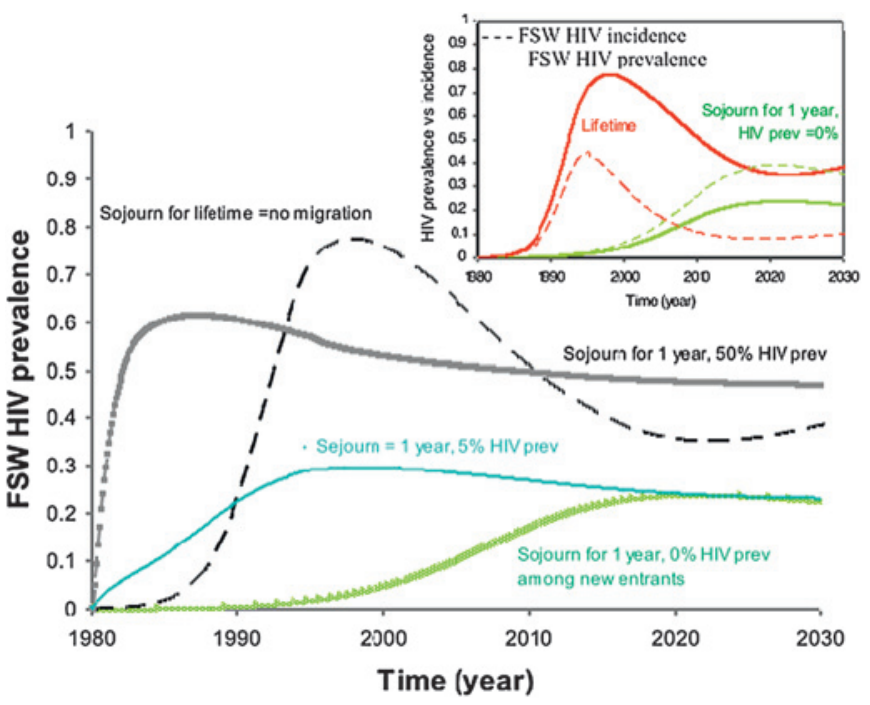

Abstract P1-S2.04 Figure 1 Cotonou.

Conclusions The impact of FSW turnover on HIV is complex but relatively modest if occurring at a slower rate than 0.20 per person-year (ie 1/5 years). Monitoring change in FSW turnover is important to interpret HIV prevalence trends over time, especially following an HIV intervention. Targeted FSW intervention remains effective in presence of rapid turn-over if new FSW can be reached rapidly.

\section{P1-S2.05 CLIENTS OF STREET-BASED FEMALE SEX WORKERS AND POTENTIAL BRIDGING OF HIV/STI IN RUSSIA}

doi:10.1136/sextrans-2011-050108.62

${ }^{1} \mathrm{~L}$ Niccolai, ${ }^{2} \mathrm{~V}$ Odinokova, ${ }^{2} \mathrm{~L}$ Safiullina, ${ }^{2} \mathrm{Z}$ Bodanovskaya, ${ }^{1} \mathrm{R}$ Heimer, ${ }^{2} \mathrm{O}$ Levina, ${ }^{2}$ M Rusakova. ${ }^{1}$ Yale School of Public Health, New Haven, USA; ${ }^{2}$ NGO Stellit, Russian Federation

Background Russia is currently experiencing one of the fastest growing HIV epidemics in the world. Transmission is concentrated among injection drug users and female sex workers (FSW), but sexual transmission to the general population is increasing. The prevalence of HIV among street-based FSW in St. Petersburg, many of whom use drugs, was $48 \%$ in 2003, but the role of their male clients in HIV transmission and bridging to the general population has yet to be studied.

Methods In a pilot study to determine feasibility of involving clients in a research study and describe HIV risk behaviours, we interviewed 62 clients during February-March of 2010 in St. Petersburg Russia. Recruitment methods included FSW and peer referrals and street intercept. Behavioural data collection covered condom use with different types of sex partners, substance use, and STI/HIV testing histories.

Results A majority of clients (74\%) reported having non-FSW partners during the past 12 months, and nearly half of the total sample $(47 \%)$ reported having regular non-FSW sex partners. Inconsistent condom use was reported by $39 \%$ of clients with FSW partners and $57 \%$ with their non-FSW partners. A majority of clients (58\%) was classified as active or potential bridgers based on having both FSW and non-FSW partners and reporting inconsistent condom use with non-FSW partners. A majority (61\%) also reported concurrent FSW and non-FSW partners. Nearly half (48\%) of last reported sex contacts with FSW involved consumption of alcohol by the client. Non-injection and injection drug use in the past

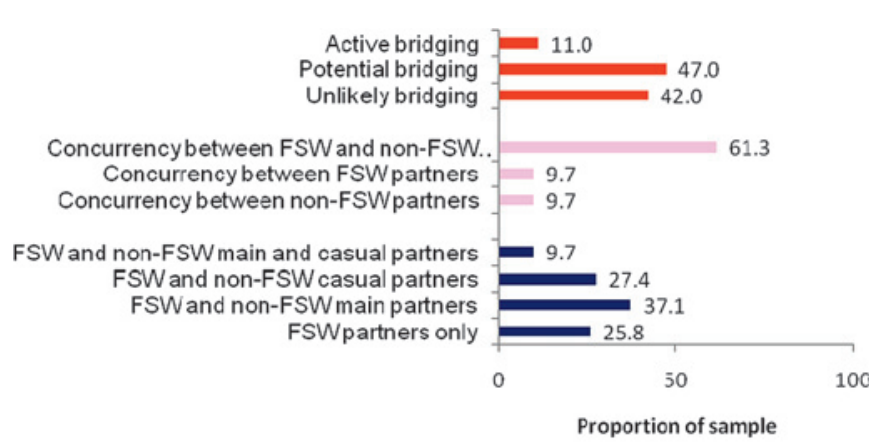

Bridging status $\square$ Concurrency status $\square$ Partner mix

Abstract P1-S2.05 Figure 1 Distributions of bridging status* concurrency, and partner mix in the past 12 months among clients of street-based FSW in St. Petersburg, Russia $(n=62)$. *Active bridginginconsistent condom use during vaginal sex with both FSW and non-FSW partners; potential bridging-consistent condom use during vaginal sex with FSW partners and inconsistent condom use during vaginal sex with non-FSW partners; unlikely bridging - consistent condom use during vaginal sex with FSW and non-FSW partners, or consistent condom use during vaginal sex with FSW and not having a non-FSW sex partner. 
30 days were reported by $15 \%$ and $7 \%$ of clients, respectively. Reported history of an STI was also common at $29 \%$. A previous HIV test was reported by 74\%; active/potential bridgers were significantly less likely than unlikely bridgers to have ever been tested for HIV.

Conclusions Results of this pilot study demonstrate the feasibility of successfully recruiting clients of FSW in a setting of epidemic growth and high HIV prevalence among FSW. A majority of clients of FSW also have non-FSW sex partners, and these partnerships are often concurrent. Condom use is sub-optimal with both FSW and non-FSW partners, and drug and alcohol use were common. These risk behaviours signal potential for HIV/STI transmission among male clients of FSW in St. Petersburg Russia, and indicate a pressing need to better understand the context and nature of risk for this potentially important bridging population. participants). Bridge clients were significantly less educated, more employed, paid lower prices to the FSW just visited, and had a previous STI.

Conclusions Clients showed high-risk sexual behaviour, a relatively high prevalence of HIV in clients compared to national estimates and a substantial proportion of them act as a bridge for HIV/STI transmission between FSW and the general population in Escuintla. Given that this is fuelling the current HIV epidemic, preventive interventions addressing this hard-to-reach group are urgently required. A significant challenge is to overcome the difficulty of reaching clients as well as to address the variety of high-risk behaviours among clients by partner type in this setting and take into account traditional concepts of masculinity.

\section{P1-S2.07 STI RATES AND RISK FACTORS AMONG FEMALE SEX WORKERS ATTENDING STI TESTING SITES IN GERMANY, JANUARY-DECEMBER 2010}

doi:10.1136/sextrans-2011-050108.64 BEHAVIOURS AMONG CLIENTS OF SEX WORKERS IN GUATEMALA - ARE THEY A BRIDGE IN HIV TRANSIMISSION?

doi:10.1136/sextrans-2011-050108.63

\begin{abstract}
${ }^{1,2} \mathrm{M}$ Sabidó, ${ }^{3,4} \mathrm{M}$ Lahuerta, ${ }^{5} \mathrm{G}$ Hernández, ${ }^{2,6} \mathrm{~A}$ Montoliu, ${ }^{2,6} \mathrm{~V}$ Gonzalez, ${ }^{7} \mathrm{~F}$ Giardina, ${ }^{5} \mathrm{~J}$ E Monzón, ${ }^{8} \mathrm{M}$ I Pedroza, ${ }^{2,6} \mathrm{~J}$ Casabona, ${ }^{8} \mathrm{R}$ Guevara. ${ }^{1}$ Fundació Sida i Societat, Barcelona, Catalonia, Spain; ${ }^{2}$ CIBER Epidemiología y Salud Pública (CIBERESP), Barcelona, Spain; ${ }^{3}$ International Center for AIDS Care and Treatment Programs, Columbia University-Mailman School of Public Health, New York, USA; ${ }^{4}$ Fundació Sida i Societat, Barcelona, USA; ${ }^{5}$ Fundació Sida i Societat, Escuintla, Guatemala, Guatemala; ${ }^{6}$ Centre for Epidemiological Studies on HIVIAIDS and STI of Catalonia (CEEISCAT), ICO/Health Department, Generalitat de Catalunya, Badalona, Spain; ${ }^{7}$ Fundació Sida i Societat, Barcelona, Catalonia, Spain, Spain; ${ }^{8}$ Health District of Escuintla, Ministry of Health, Guatemala, Guatemala
\end{abstract}

Background Clients of female sex workers (FSW) are an important target group for HIV/STI prevention. This study aimed to estimate the prevalence of HIV and other sexually transmitted infections (STI) prevalence in clients examine their risk behaviours and evaluate their role as a bridge population in the spread of HIV/STI.

Methods Cross-sectional study conducted between 2008 and 2009 among 553 clients recruited through a two-stage convenient sample in commercial sex sites in the province of Escuintla, Guatemala. They were interviewed and tested for HIV and other STI. Bridge clients were defined as those who engaged in sex with both regular partners and FSWs but reported inconsistent condom use with FSWs whereas potential bridge as those reported consistent condom use with FSWs. Non-bridge clients engaged in sex with only FSWs. Multinomial and multivariable logistic regression models were used to identify risk factors associated with the bridge category and with having $\geq 1$ STI/HIV, respectively.

Results Half of the clients approached refused participation. Median age was 28.9 years; $57.7 \%$ had a regular partner, of whom $10.1 \%$ had concurrent non-commercial partnerships. Consistent condom use with FSW and regular partners was $72.5 \%$ and $17.1 \%$, respectively. The main reason for not using condoms was trust (49.5\%). Approximately $18 \%$ formed a bridge, and $40.0 \%$ a potential bridge. Among those who provided samples $(70.5 \%$ a blood sample and $89.7 \%$ urine sample), prevalence of HIV, syphilis, gonorrhoea, chlamydia, and herpes simplex virus 2 was $1.5 \%, 1.0 \%, 0.8 \%, 5.5 \%$, and $3.4 \%$, respectively. Unprotected sex with FSWs and drug use just before sex were risk factors for having any STI $(9.8 \%$ of 